\title{
The tractability index of memristive circuits: branch-oriented and tree-based models ${ }^{* \dagger}$
}

\author{
FERNANDo García-REDONDo ${ }^{\ddagger}$ and RICARdo RiazA ${ }^{\S}$ \\ Departamento de Matemática Aplicada a las Tecnologías de la Información \\ Escuela Técnica Superior de Ingenieros de Telecomunicación \\ Universidad Politécnica de Madrid - 28040 Madrid, Spain
}

\begin{abstract}
The memory-resistor or memristor is a new electrical element characterized by a nonlinear charge-flux relation. This device poses many challenging problems, in particular from the circuit modeling point of view. In this paper we address the index analysis of certain differential-algebraic models of memristive circuits; specifically, our attention is focused on so-called branch-oriented models, which include in particular tree-based formulations of the circuit equations. Our approach combines results coming from DAE theory, matrix analysis and the theory of digraphs. This framework should be useful in future studies of dynamical aspects of memristive circuits.
\end{abstract}

Keywords: nonlinear circuit, memristor, differential-algebraic equation, semistate model, index, tree.

AMS subject classification: 05C50, 15A22, 34A09, 94C05, 94C15.

\section{Introduction}

The memory-resistor or memristor is considered as the fourth basic element in circuit theory, besides resistors, inductors and capacitors. It is defined by a nonlinear charge-flux characteristic, which may have either a charge-controlled form $\varphi=\phi(q)$ or a flux-controlled one $q=\sigma(\varphi)$. The existence of such a device was already postulated for symmetry reasons by Chua in 1971 [9] (see also [11]), and the actual appearance of memristors in nanoscale electronics announced in [40] has raised a renewed interest in these devices. A lot of analytical

\footnotetext{
*Supported by Research Projects MTM2007-62064 of Ministerio de Educación y Ciencia, Spain, MTM2010-15102 of Ministerio de Ciencia e Innovacion, and CCG10-UPM/ESP-5236 of Comunidad de Madrid/UPM, Spain.

${ }^{\dagger}$ This is a pre-peer reviewed version of the following article: F. García-Redondo and R. Riaza, The tractability index of memristive circuits: branch-oriented and tree-based models, Mathematical Methods in the Applied Sciences, accepted for publication, 2012.

${ }^{\ddagger}$ E-mail: fernando.garcia.redondo@alumnos.upm.es.

${ }^{\S}$ Corresponding author. E-mail: ricardo.riaza@upm.es.
} 
aspects of memristors and memristive-based circuits, as well as many applications, have been reported in the last two years; cf. [3, 8, 21, 22, 28, 29, 30, 35, 37, 39, 45, 46] and references therein.

Modeling and analyzing memristive circuits pose many challenging mathematical problems. The nonlinear character of memristors naturally leads to the use of time-domain circuit models. These models may be state-space ones, based on explicit ordinary differential equations (ODEs); nevertheless, in the presence of a high number of devices, the circuit equations are usually set up in terms of semistate models based on differential-algebraic equations (DAEs) [12, 17, 18, 20, 34, 42, 43]. As detailed below, the analysis of memristive circuit models involves in turn a systematic use of digraph theory and matrix analysis, including matrix pencil theory. This drives the study of modeling aspects of memristive circuits to an interdisciplinary framework.

In this paper we undertake the analysis of the DAE index of the memristive circuit models arising from the so-called branch-oriented approach. These models are closely related to treebased systems and hybrid analysis [20, 33, 41], and play a key role in the study of many analytical features of circuit theory, not only to set up the networks equations but also to address different problems which include, among others, the state formulation problem and several dynamic properties, including stability aspects [34, 38].

The above-mentioned index of a differential-algebraic circuit model can be introduced in different ways. The reader is referred to $[5,15,19,23,25,31,34]$ for detailed discussions of the different index notions, which include the differentiation, geometric, perturbation, strangeness and tractability indices. The tractability index concept will be of particular interest and is presented in Section 3. All the index concepts can be understood to generalize the Weierstrass-Kronecker index of a matrix pencil [14] to time-varying and/or nonlinear settings. Within the circuit context, the tractability index of different DAE models of classical circuits does not exceed two under passivity assumptions [12, 17, 18, 33, 34, 42, 43]; index one configurations are of special interest, because they allow for the use of efficient theoretical and numerical tools in their analysis. Some of these results have been recently extended to nodal models of memristive circuits [37]. In the present paper we address the index analysis of a different family of circuit models, which arise from a branch-oriented approach and require different graph-theoretic techniques. Our results will apply in particular to tree-based formulations, very often used in practice.

The paper is structured as follows. Section 2 compiles some background on digraph theory and also on memristive circuits. Section 3 introduces the tractability index notion, which supports the index analysis presented in Section 4. Some concluding remarks can be found in Section 5 . 


\section{Background}

\subsection{Some results from digraph theory}

Some background on digraph theory is necessary before introducing the circuit models to be analyzed throughout the paper. Specifically, we will make systematic use of the loop and cutset matrices introduced below and, occasionally, of the so-called fundamental loop and cutset matrices arising from the choice of a spanning tree.

Consider a directed graph with $n$ nodes, $m$ branches and $k$ connected components. Chosen an orientation in every loop, the loop matrix $\tilde{B}$ is defined as $\left(b_{i j}\right)$, where

$$
b_{i j}=\left\{\begin{aligned}
1 & \text { if branch } j \text { is in loop } i \text { with the same orientation } \\
-1 & \text { if branch } j \text { is in loop } i \text { with the opposite orientation } \\
0 & \text { if branch } j \text { is not in loop } i
\end{aligned}\right.
$$

The rank of this matrix can be shown to equal $m-n+k$. A reduced loop matrix $B$ is any $((m-n+k) \times m)$-submatrix of $\tilde{B}$ with full row rank.

A subset $K$ of the set of branches of a digraph is a cutset if the removal of $K$ increases the number of connected components of the digraph, and it is minimal with respect to this property, that is, the removal of any proper subset of $K$ does not increase the number of components. In a connected digraph, a cutset is just a minimal disconnecting set of branches. The removal of the branches of a cutset increases the number of connected components by exactly one. Furthermore, all the branches of a cutset may be shown to connect the same pair of connected components of the digraph which results from the deletion of the cutset. This makes it possible to define the orientation of a cutset, say from one of these components towards the other. The cutset matrix $\tilde{D}=\left(d_{i j}\right)$ is then defined by

$$
d_{i j}=\left\{\begin{aligned}
1 & \text { if branch } j \text { is in cutset } i \text { with the same orientation } \\
-1 & \text { if branch } j \text { is in cutset } i \text { with the opposite orientation } \\
0 & \text { if branch } j \text { is not in cutset } i
\end{aligned}\right.
$$

The rank of $\tilde{D}$ can be proved to be $n-k$; any set of $n-k$ linearly independent rows of $\tilde{D}$ defines a reduced cutset matrix $D \in \mathbb{R}^{(n-k) \times m}$. In a connected digraph, any reduced cutset matrix has order $(n-1) \times m$.

Certain submatrices of $B$ and $D$ characterize the existence of so-called $K$-cutsets and $K$ loops (that is, cutsets or loops just defined by branches belonging to a given set of branches $K)$, as stated below. We denote by $B_{K}\left(\right.$ resp. $\left.B_{\mathcal{G}-K}\right)$ the submatrix of $B$ defined by the columns which belong (resp. do not belong) to $K$; the same applies to the cutset matrix $D$. The reader is referred to [34, Sect. 5.1] for explicit proofs of the following assertions.

Lemma 1. Let $K$ be a subset of branches of a given digraph $\mathcal{G}$. The following assertions are equivalent:

(a) $K$ does not contain cutsets; 
(b) $B_{K}$ has full column rank;

(c) $D_{\mathcal{G}-K}$ has full row rank.

Analogously, the following statements are equivalent:

(d) $K$ does not contain loops;

(e) $D_{K}$ has full column rank;

(f) $B_{\mathcal{G}-K}$ has full row rank.

The following digraph analog of Tellegen's theorem will also be useful in our analysis (see e.g. [13] (Section 7.4)).

Lemma 2. If the columns of the reduced loop and cutset matrices $B, D$ of a digraph are arranged according to the same order of branches, then $B D^{T}=0, D B^{T}=0$.

Actually, the relations $\operatorname{im} D^{T}=\operatorname{ker} B$ and $\operatorname{im} B^{T}=\operatorname{ker} D$ hold true. Hence, the cut space im $D^{T}$ spanned by the rows of $D$ can be described as $\operatorname{ker} B$ and, analogously, the cycle space $\operatorname{im} B^{T}$ spanned by the rows of $B$ equals ker $D$ [4]. These spaces are orthogonal to each other since $\left(\operatorname{im} D^{T}\right)^{\perp}=(\operatorname{ker} B)^{\perp}=\operatorname{im} B^{T}$.

A particular form of the loop and cutset matrices follows from the choice of a spanning tree in the digraph (see e.g. [10, 34]). The branches in the tree are called twigs, whereas the remaining ones are called links. A well-known property in digraph theory states that every link defines a unique loop together with some twigs, and every twig defines a unique cutset together with some links. Because of this property and Lemma 2, the so-called fundamental loop and cutset matrices constructed from a given tree read as

$$
B=\left(\begin{array}{ll}
F & I
\end{array}\right), \quad D=\left(I-F^{T}\right)
$$

for a certain submatrix $F$.

\subsection{Memristive circuits}

Consider a nonlinear, connected, time-invariant circuit composed of capacitors, inductors, resistors, memristors, and independent voltage and current sources. Capacitors and inductors will have $C^{1}$ voltage- and current-controlled characteristics $q_{c}=\psi\left(v_{c}\right), \varphi_{l}=\eta\left(i_{l}\right)$, respectively; we denote by $C\left(v_{c}\right)$ and $L\left(i_{l}\right)$ the incremental capacitance and inductance matrices $\psi^{\prime}\left(v_{c}\right), \eta^{\prime}\left(i_{l}\right)$.

Memristors will be defined by the $C^{2}$ charge-controlled relation $\varphi_{m}=\phi\left(q_{m}\right)$. The incremental memristance is $M\left(q_{m}\right)=\phi^{\prime}\left(q_{m}\right)$. Note that the relations $\varphi_{m}^{\prime}(t)=v_{m}(t), q_{m}^{\prime}(t)=i_{m}(t)$ yield $v_{m}(t)=M\left(q_{m}(t)\right) i_{m}(t)$; the "memory-resistor" name comes from the fact that the device behaves as a resistor in which the resistance depends on $q_{m}(t)=\int_{-\infty}^{t} i_{m}(\tau) d \tau$. Resistors will be assumed to be current-controlled by a $C^{1}$ map of the form $v_{r}=\rho\left(i_{r}\right)$, and we let $R\left(i_{r}\right)$ stand for the incremental resistance matrix $\rho^{\prime}\left(i_{r}\right)$. Under a strict passivity assumption on resistors and memristors, the results will be shown to hold also in the presence of 
voltage-controlled resistors (note that the nonlinear nature allowed for all circuit elements makes e.g. a diode belong to this class of devices) and/or flux-controlled memristors. Recall that a given set of devices (capacitors, inductors, resistors or memristors) are strictly passive if the corresponding matrix $P$ (standing for $C\left(v_{c}\right), L\left(i_{l}\right), R\left(i_{r}\right)$ or $M\left(q_{m}\right)$, respectively) is positive definite, that is, it verifies $u^{T} P u>0$ for any non-vanishing vector $u$.

Writing the excitation terms coming from the voltage and current sources, respectively, as $v_{s}(t), i_{s}(t)$, the circuit equations can be written in the form

$$
\begin{aligned}
C\left(v_{c}\right) v_{c}^{\prime} & =i_{c} \\
L\left(i_{l}\right) i_{l}^{\prime} & =v_{l} \\
q_{m}^{\prime} & =i_{m} \\
0 & =v_{m}-M\left(q_{m}\right) i_{m} \\
0 & =v_{r}-\rho\left(i_{r}\right) \\
0 & =B_{c} v_{c}+B_{l} v_{l}+B_{m} v_{m}+B_{r} v_{r}+B_{u} v_{s}(t)+B_{j} v_{j} \\
0 & =D_{c} i_{c}+D_{l} i_{l}+D_{m} i_{m}+D_{r} i_{r}+D_{u} i_{u}+D_{j} i_{s}(t),
\end{aligned}
$$

where we are expressing Kirchhoff laws in terms of the loop and cutset matrices as $B v=0$, $D i=0$. Note that the loop matrix $B$ is split as $\left(B_{c} B_{l} B_{m} B_{r} B_{u} B_{j}\right)$, where $B_{c}$ (resp. $\left.B_{l}, B_{m}, B_{r}, B_{u}, B_{j}\right)$ corresponds to the columns accommodating capacitors (resp. inductors, memristors, resistors, voltage sources, current sources). The same applies to the cutset matrix $D$.

It is worth emphasizing that system (1) covers, in particular, models coming from the choice of a given spanning tree in the circuit; these tree-based models are very often used in

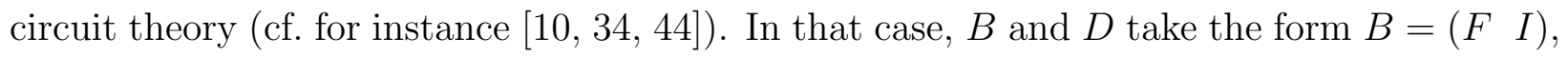
$D=\left(I-F^{T}\right)$; this means that the voltages of link devices are expressed in terms of twig voltages in (1f) and, analogously, twig currents are written in terms of link currents in (1g). The results will therefore apply in particular to such tree-based models. In our analysis we will not make use of the special form of these fundamental matrices, though.

The circuit model (1) has the form of a quasilinear (or linearly implicit) differentialalgebraic equation (DAE), namely,

$$
A(x) x^{\prime}=g(x, t)
$$

where $A$ stands for a block-diagonal matrix block-diag $(C, L, I, 0)$ and $x$ comprises all the branch variables entering the model. The right-hand side can be actually written as

$$
g(x, t)=f(x)+s(t)
$$

because the excitation terms $B_{u} v_{s}(t), D_{j} i_{s}(t)$ coming from the voltage and current sources are decoupled from the remaining terms. Section 3 provides some general background on DAEs and their index; we will then tackle, in Section 4, the characterization of the tractability index of (1) in the light of its linearly implicit form (2). 
In the sequel, all circuits will be assumed to be well-posed; this means that V-loops (loops just defined by voltage sources) and I-cutsets (cutset defined by current sources only) will be precluded. The same terminological convention will be used in the index analysis of Section 4, where a $V C$-loop will stand for a loop defined just by voltage sources and/or capacitors, and an IL-cutset will be a cutset including only current sources and/or inductors.

\section{Projector-based analysis of DAE circuit models}

Differential-algebraic equations, also known as semistate systems, constrained equations or descriptor systems, arise in different application fields; in addition to circuit theory, these include mechanics, control theory, power systems theory, and others [5, 15, 19, 23, 25, 31, 34]. Many analytical and numerical properties of DAEs rely on their index: we present in this section the tractability index concept and some projector-based results which will be useful in the computation of this index.

The characterization of the tractability index of a given DAE model is of major importance, not only because it paves the way for an appropriate numerical treatment in simulation, but also because it reduces the description of the dynamical behavior to that of an inherent explicit ODE; this is performed by means of a decoupling of the different solution components $[25,34,43]$. Note that the tractability index has been already proved to be a very useful tool in circuit theory, specially regarding the analysis of Modified Nodal Analysis (MNA) models [12, 27, 34, 42, 43]. See also [17, 18, 33, 36]; related results can be found in $[32,41]$. The discussion of the tractability index notion will be restricted to cases with index not greater than two; this is due to the fact that the index of DAEs modeling a very large class of electrical and electronic circuits does not exceed two [12, 33, 34, 42]. This way we avoid certain difficulties exhibited in problems with arbitrary index.

Let us first remark that the kernel of $A(x)$ in (2) is constant, provided that the capacitance and inductance matrices $C\left(v_{c}\right), L\left(i_{l}\right)$ in (1) are non-singular. Letting $B(x)$ stand for the Jacobian matrix $-f^{\prime}(x)$ (cf. (3)), and denoting by $Q$ a constant projector onto ker $A(x)$ (so that $Q^{2}=Q$ with $\operatorname{im} Q=\operatorname{ker} A(x)$ ), the DAE (2) has tractability index one if the matrix $A_{1}(x)=A(x)+B(x) Q$ is non-singular. By contrast, consider a setting in which $A_{1}(x)$ is rank-deficient everywhere, in a way such that there exists a continuous projector $Q_{1}(x)$ onto ker $A_{1}(x)$ (forcing $A_{1}(x)$ to have constant rank). Let $B_{1}(x)$ stand for the product $B(x)(I-Q)$. Basing on the special form of the circuit equations (cf. [43, Remark A.18]), system (2) will be said to have tractability index two if $A_{2}(x)=A_{1}(x)+B_{1}(x) Q_{1}(x)$ is non-singular. This definition of the index is simpler than the one for general nonlinear DAEs $[25,43]$.

In a linear time-invariant setting, the analysis of DAEs can be performed in terms of the associated matrix pencil; cf. [14]. For time-varying and/or linearly implicit problems the relation with matrix pencil theory is more involved [5, 31, 34]. In particular, the relation between the tractability index of (2) and the Weierstrass-Kronecker index of the matrix pencil $\left\{A\left(x^{*}\right), B\left(x^{*}\right)\right\}$ arising from the linearized problem were thoroughly examined in $[15,16,24]$ (recent related results can be found in $[25,26]$ ). In Section 4 we will make use of 
the fact that a matrix pencil $\{A, B\}$ with singular $A$ is regular with Weierstrass-Kronecker index one if and only if the matrix $A_{1}=A+B Q$ is non-singular, $Q$ being any projector onto ker $A$. Additionally, if $A_{1}$ is singular, the pencil can be shown to be regular with WeierstrassKronecker index two if and only if $A_{2}=A_{1}+B_{1} Q_{1}$ is non-singular, where $Q_{1}$ is any projector onto ker $A_{1}$ and $B_{1}=B(I-Q)$. The index analysis of the circuit model (1) will be crucially supported on these results.

\section{The index of branch-oriented models of memristive circuits}

The main result of this paper is the tractability index characterization of branch-oriented circuit models stated in Theorem 1 below. From a mathematical point of view, the analysis will be based on the results compiled in subsection 2.1 and Section 3.

\subsection{Index analysis of the branch-oriented model}

Theorem 1. Consider a well-posed circuit in which the capacitance and inductance matrices $C\left(v_{c}\right), L\left(i_{l}\right)$ are non-singular, and the resistance and memristance matrices $R\left(i_{r}\right), M\left(q_{m}\right)$ are positive definite. The following assertions hold.

(i) The model (1) has tractability index one if and only if the circuit has neither VC-loops nor IL-cutsets.

(ii) Suppose, additionally, that $C\left(v_{c}\right), L\left(i_{l}\right)$ are positive definite. In the presence of $V C$ loops and/or IL-cutsets, system (1) has tractability index two.

Proof. The leading matrix $A(x)$ has the structure block-diag $\left(C\left(v_{c}\right), L\left(i_{l}\right), I_{m}, 0\right)$, whereas $B(x)$ can be written as

$$
B(x)=\left(\begin{array}{ccccccccccc}
0 & 0 & 0 & -I_{c} & 0 & 0 & 0 & 0 & 0 & 0 & 0 \\
0 & 0 & 0 & 0 & -I_{l} & 0 & 0 & 0 & 0 & 0 & 0 \\
0 & 0 & 0 & 0 & 0 & -I_{m} & 0 & 0 & 0 & 0 & 0 \\
0 & 0 & \frac{\partial M\left(q_{m}\right) i_{m}}{\partial q_{m}} & 0 & 0 & -M\left(q_{m}\right) & I_{m} & 0 & 0 & 0 & 0 \\
0 & 0 & 0 & 0 & 0 & 0 & 0 & -R\left(i_{r}\right) & I_{r} & 0 & 0 \\
B_{c} & 0 & 0 & 0 & B_{l} & 0 & B_{m} & 0 & B_{r} & 0 & B_{j} \\
0 & D_{l} & 0 & D_{c} & 0 & D_{m} & 0 & D_{r} & 0 & D_{u} & 0
\end{array}\right) .
$$

Note that we have changed the sign of (1d)-(1g) for notational simplicity. A projector $Q$ onto ker $A(x)$ with the structure block-diag $(0, I)$ confers $A_{1}(x)=A(x)+B(x) Q$ the form

$$
A_{1}(x)=\left(\begin{array}{ccccccccccc}
C\left(v_{c}\right) & 0 & 0 & -I_{c} & 0 & 0 & 0 & 0 & 0 & 0 & 0 \\
0 & L\left(i_{l}\right) & 0 & 0 & -I_{l} & 0 & 0 & 0 & 0 & 0 & 0 \\
0 & 0 & I_{m} & 0 & 0 & -I_{m} & 0 & 0 & 0 & 0 & 0 \\
0 & 0 & 0 & 0 & 0 & -M\left(q_{m}\right) & I_{m} & 0 & 0 & 0 & 0 \\
0 & 0 & 0 & 0 & 0 & 0 & 0 & -R\left(i_{r}\right) & I_{r} & 0 & 0 \\
0 & 0 & 0 & 0 & B_{l} & 0 & B_{m} & 0 & B_{r} & 0 & B_{j} \\
0 & 0 & 0 & D_{c} & 0 & D_{m} & 0 & D_{r} & 0 & D_{u} & 0
\end{array}\right) .
$$


Proof of (i). System (1) is index one if and only if the matrix (5) is non-singular, a condition which relies on the non-singularity of

$$
\left(\begin{array}{cccccccc}
0 & 0 & -M\left(q_{m}\right) & I_{m} & 0 & 0 & 0 & 0 \\
0 & 0 & 0 & 0 & -R\left(i_{r}\right) & I_{r} & 0 & 0 \\
0 & B_{l} & 0 & B_{m} & 0 & B_{r} & 0 & B_{j} \\
D_{c} & 0 & D_{m} & 0 & D_{r} & 0 & D_{u} & 0
\end{array}\right)
$$

and in turn this matrix is non-singular if and only if so it is

$$
\left(\begin{array}{cccccc}
0 & B_{l} & B_{m} M\left(q_{m}\right) & B_{r} R\left(i_{r}\right) & 0 & B_{j} \\
D_{c} & 0 & D_{m} & D_{r} & D_{u} & 0
\end{array}\right) .
$$

In order to prove the index one claim we then need to show that (7) is non-singular if and only if the circuit has neither VC-loops nor IL-cutsets. Clearly this is a necessary condition, since otherwise according to Lemma 1 (items (b) and (e)) either $\left(D_{c} D_{u}\right)$ or $\left(B_{l} B_{j}\right)$ would not have full column rank.

Conversely, assume that there are neither VC-loops nor IL-cutsets and suppose that a vector $\left(x^{T} y^{T}\right)$ belongs to the left-kernel of (7), that is, suppose that

$$
\begin{aligned}
y^{T} D_{c} & =0 \\
x^{T} B_{l} & =0 \\
x^{T} B_{m} M\left(q_{m}\right)+y^{T} D_{m} & =0 \\
x^{T} B_{r} R\left(i_{r}\right)+y^{T} D_{r} & =0 \\
y^{T} D_{u} & =0 \\
x^{T} B_{j} & =0 .
\end{aligned}
$$

At this point we make use of the identity

$$
B_{c} D_{c}^{T}+B_{l} D_{l}^{T}+B_{m} D_{m}^{T}+B_{r} D_{r}^{T}+B_{u} D_{u}^{T}+B_{j} D_{j}^{T}=0,
$$

which follows from Lemma 2. Multiplying (9) from the left by $x^{T}$ and from the right by $y$, and using (8a), (8b), (8e) and (8f), we get

$$
x^{T} B_{m} D_{m}^{T} y+x^{T} B_{r} D_{r}^{T} y=0 .
$$

Additionally, using (8c) and (8d) this equation can be rewritten as

$$
x^{T} B_{m} M\left(q_{m}\right)^{T} B_{m}^{T} x+x^{T} B_{r} R\left(i_{r}\right)^{T} B_{r}^{T} x=0 .
$$

Using the fact that $M^{T}$ and $R^{T}$ are positive definite since so they are $M$ and $R$, from (10) we get

$$
\begin{aligned}
x^{T} B_{m} & =0 \\
x^{T} B_{r} & =0 .
\end{aligned}
$$


The relations depicted in (8b), (8f), (11a) and (11b), together with the equivalence of items (d) and (f) in Lemma 1 and the absence of VC-loops, imply that $x=0$.

Similarly, the insertion of (11a) and (11b) into (8c) and (8d) leads to

$$
\begin{aligned}
y^{T} D_{m} & =0 \\
y^{T} D_{r} & =0
\end{aligned}
$$

which, together with (8a) and (8e), the equivalence of items (a) and (c) in Lemma 1, and the absence of IL-cutsets, yield $y=0$. This means that (7) is indeed non-singular and, hence, that (1) is index one in the absence of VC-loops and IL-cutsets.

Proof of (ii). Assume now that the circuit displays VC-loops and/or IL-cutsets. Due to items (b) and (e) of Lemma 1 this implies that $\left(D_{c} D_{u}\right)$ and/or $\left(B_{l} B_{j}\right)$ do not have full column rank, making (6) -and hence the $A_{1}$ matrix in (5)- singular.

We denote by $\hat{Q}$ a projector onto $\operatorname{ker}\left(D_{c} D_{u}\right)$. Note that $\hat{Q}=0$ in the absence of VCloops. Analogously, $\tilde{Q}$ will stand for a projector onto $\operatorname{ker}\left(B_{l} B_{j}\right)$, with $\tilde{Q}=0$ in problems without IL-cutsets; keep in mind, though, that in the setting of item (ii) both projectors cannot vanish simultaneously. Splitting $\hat{Q}$ and $\tilde{Q}$ in the form

$$
\hat{Q}=\left(\begin{array}{ll}
\hat{Q}_{11} & \hat{Q}_{12} \\
\hat{Q}_{21} & \hat{Q}_{22}
\end{array}\right), \quad \tilde{Q}=\left(\begin{array}{ll}
\tilde{Q}_{11} & \tilde{Q}_{12} \\
\tilde{Q}_{21} & \tilde{Q}_{22}
\end{array}\right),
$$

a projector onto $A_{1}(x)$ can be checked to be

$$
Q_{1}=\left(\begin{array}{ccccccccccc}
0 & 0 & 0 & C^{-1} \hat{Q}_{11} & 0 & 0 & 0 & 0 & 0 & C^{-1} \hat{Q}_{12} & 0 \\
0 & 0 & 0 & 0 & L^{-1} \tilde{Q}_{11} & 0 & 0 & 0 & 0 & 0 & L^{-1} \tilde{Q}_{12} \\
0 & 0 & 0 & 0 & 0 & 0 & 0 & 0 & 0 & 0 & 0 \\
0 & 0 & 0 & \hat{Q}_{11} & 0 & 0 & 0 & 0 & 0 & \hat{Q}_{12} & 0 \\
0 & 0 & 0 & 0 & \tilde{Q}_{11} & 0 & 0 & 0 & 0 & 0 & \tilde{Q}_{12} \\
0 & 0 & 0 & 0 & 0 & 0 & 0 & 0 & 0 & 0 & 0 \\
0 & 0 & 0 & 0 & 0 & 0 & 0 & 0 & 0 & 0 & 0 \\
0 & 0 & 0 & 0 & 0 & 0 & 0 & 0 & 0 & 0 & 0 \\
0 & 0 & 0 & 0 & 0 & 0 & 0 & 0 & 0 & 0 & 0 \\
0 & 0 & 0 & \hat{Q}_{21} & 0 & 0 & 0 & 0 & 0 & \hat{Q}_{22} & 0 \\
0 & 0 & 0 & 0 & \tilde{Q}_{21} & 0 & 0 & 0 & 0 & 0 & \tilde{Q}_{22}
\end{array}\right) .
$$

Some easy computations show that the matrix $A_{2}(x)$ has the expression

$$
\left(\begin{array}{ccccccccccc}
C\left(v_{c}\right) & 0 & 0 & -I_{c} & 0 & 0 & 0 & 0 & 0 & 0 & 0 \\
0 & L\left(i_{l}\right) & 0 & 0 & -I_{l} & 0 & 0 & 0 & 0 & 0 & 0 \\
0 & 0 & I_{m} & 0 & 0 & -I_{m} & 0 & 0 & 0 & 0 & 0 \\
0 & 0 & 0 & 0 & 0 & -M\left(q_{m}\right) & I_{m} & 0 & 0 & 0 & 0 \\
0 & 0 & 0 & 0 & 0 & 0 & 0 & -R\left(i_{r}\right) & I_{r} & 0 & 0 \\
0 & 0 & 0 & B_{c} C^{-1} \hat{Q}_{11} & B_{l} & 0 & B_{m} & 0 & B_{r} & B_{c} C^{-1} \hat{Q}_{12} & B_{j} \\
0 & 0 & 0 & D_{c} & D_{l} L^{-1} \tilde{Q}_{11} & D_{m} & 0 & D_{r} & 0 & D_{u} & D_{l} L^{-1} \tilde{Q}_{12}
\end{array}\right)
$$


and its non-singularity relies on that of

$$
\left(\begin{array}{cccccccc}
0 & 0 & -M\left(q_{m}\right) & I_{m} & 0 & 0 & 0 & 0 \\
0 & 0 & 0 & 0 & -R\left(i_{r}\right) & I_{r} & 0 & 0 \\
B_{c} C^{-1} \hat{Q}_{11} & B_{l} & 0 & B_{m} & 0 & B_{r} & B_{c} C^{-1} \hat{Q}_{12} & B_{j} \\
D_{c} & D_{l} L^{-1} \tilde{Q}_{11} & D_{m} & 0 & D_{r} & 0 & D_{u} & D_{l} L^{-1} \tilde{Q}_{12}
\end{array}\right)
$$

In turn, this matrix is non-singular if and only if so it is

$$
\left(\begin{array}{cccccc}
B_{c} C^{-1} \hat{Q}_{11} & B_{l} & B_{m} M\left(q_{m}\right) & B_{r} R\left(i_{r}\right) & B_{c} C^{-1} \hat{Q}_{12} & B_{j} \\
D_{c} & D_{l} L^{-1} \tilde{Q}_{11} & D_{m} & D_{r} & D_{u} & D_{l} L^{-1} \tilde{Q}_{12}
\end{array}\right)
$$

Premultiplying (15) by $\left(x^{T} y^{T}\right)$ we get the identities

$$
\begin{aligned}
x^{T} B_{c} C^{-1} \hat{Q}_{11}+y^{T} D_{c} & =0 \\
x^{T} B_{l}+y^{T} D_{l} L^{-1} \tilde{Q}_{11} & =0 \\
x^{T} B_{m} M\left(q_{m}\right)+y^{T} D_{m} & =0 \\
x^{T} B_{r} R\left(i_{r}\right)+y^{T} D_{r} & =0 \\
x^{T} B_{c} C^{-1} \hat{Q}_{12}+y^{T} D_{u} & =0 \\
x^{T} B_{j}+y^{T} D_{l} L^{-1} \tilde{Q}_{12} & =0,
\end{aligned}
$$

and we need to show that the unique solution to this system is $x=0, y=0$.

Multiply (16a) by $\hat{Q}_{11}$ and (16e) by $\hat{Q}_{21}$; adding up the resulting relations we get

$$
x^{T} B_{c} C^{-1} \hat{Q}_{11}=0
$$

where we have used the identities $\hat{Q}_{11} \hat{Q}_{11}+\hat{Q}_{12} \hat{Q}_{21}=\hat{Q}_{11}$ and $D_{c} \hat{Q}_{11}+D_{u} \hat{Q}_{21}=0$ which result from the definition of $\hat{Q}$ as a projector onto $\operatorname{ker}\left(D_{c} D_{u}\right)$. From (16a) and (17) it follows that

$$
y^{T} D_{c}=0
$$

Proceeding in exactly the same manner with the projectors $\hat{Q}_{12}$ and $\hat{Q}_{22}$ instead of $\hat{Q}_{11}$ and $\hat{Q}_{21}$ we derive

$$
x^{T} B_{c} C^{-1} \hat{Q}_{12}=0
$$

and then

$$
y^{T} D_{u}=0
$$

Analogously, working with the projector $\tilde{Q}$ onto $\operatorname{ker}\left(B_{l} B_{j}\right)$, from $(16 \mathrm{~b})$ and (16f) we get

$$
\begin{aligned}
x^{T} B_{l} & =0 \\
x^{T} B_{j} & =0 .
\end{aligned}
$$


Be aware of the fact that the identity (9) is still valid in this setting. Multiplying (9) from the left by $x^{T}$ and from the right by $y$, and using (16c), (16d), (18), (20), (21) and (22) we now derive

$$
x^{T} B_{m} M\left(q_{m}\right)^{T} B_{m}^{T} x+x^{T} B_{r} R\left(i_{r}\right)^{T} B_{r}^{T} x=0 .
$$

Due to the positive definite nature of $M$ and $R$, this implies

$$
\begin{aligned}
x^{T} B_{m} & =0 \\
x^{T} B_{r} & =0
\end{aligned}
$$

and, in the light of (16c) and (16d),

$$
\begin{aligned}
y^{T} D_{m} & =0 \\
y^{T} D_{r} & =0 .
\end{aligned}
$$

It still remains to show that the identities $x^{T} B_{c}=0$ and $y^{T} D_{l}=0$ hold true. Premultiplying (9) by $x^{T}$ and using the relations (21), (22), (24) and (25) it follows that

$$
x^{T} B_{c} D_{c}^{T}+x^{T} B_{u} D_{u}^{T}=0 .
$$

This means that

$$
\left(\begin{array}{c}
B_{c}^{T} \\
B_{u}^{T}
\end{array}\right) x \in \operatorname{ker}\left(\begin{array}{ll}
D_{c} & D_{u}
\end{array}\right)
$$

and, since $\hat{Q}$ is a projector onto $\operatorname{ker}\left(D_{c} D_{u}\right)$ and vectors on $\operatorname{im} \hat{Q}$ remain invariant, this yields

$$
\hat{Q}\left(\begin{array}{c}
B_{c}^{T} \\
B_{u}^{T}
\end{array}\right) x=\left(\begin{array}{c}
B_{c}^{T} \\
B_{u}^{T}
\end{array}\right) x,
$$

in particular

$$
\hat{Q}_{11} B_{c}^{T} x+\hat{Q}_{12} B_{u}^{T} x=B_{c}^{T} x .
$$

Now, multiplying (17) and (19) by $B_{c}^{T} x$ and $B_{u}^{T} x$, respectively, and summing up the result, we get in the light of (28)

$$
x^{T} B_{c} C^{-1} B_{c}^{T} x=0
$$

which implies

$$
x^{T} B_{c}=0
$$

because of the positive definiteness assumption on $C$, which makes $C^{-1}$ positive definite as well.

In exactly the same manner we may show that the identity

$$
y^{T} D_{l}=0
$$


holds, because of the positive definite nature of the inductance matrix $L$.

Finally, the relations (21), (22), (24), (25) and (29), together with the absence of Vloops in well-posed circuits yield, using items (d) and (f) of Lemma 1, the identity $x=0$. The relation $y=0$ follows analogously from (18), (20), (26), (27) and (30), the absence of I-cutsets and items (a) and (c) of Lemma 1. This completes the proof.

Theorem 1 extends to the context of memristive circuits the index characterization of nodal models of classical circuits discussed in $[12,42]$. Note however that the use of a branchoriented approach, which avoids the introduction of node potentials in the model, requires the use of different graph-theoretic techniques. The present characterization is shown below to hold even without a restriction on the controlling variables for resistors and memristors; in subsection 4.3 it will be also shown to apply, in particular, to tree-based models.

\subsection{Voltage-controlled resistors and flux-controlled memristors}

The assumption that resistors are current-controlled and memristors are charge-controlled is not unduly restrictive, as shown in Corollary 1 below.

Corollary 1. The index characterization stated in Theorem 1 applies to circuits with both current-and voltage-controlled resistors, as well as charge-and flux-controlled memristors, provided that all of them are strictly passive.

Indeed, assume that the set of resistors includes both current-controlled and voltagecontrolled ones. Use the subscripts 1 and 2 to distinguish both. Current-controlled resistors are governed by

$$
v_{r_{1}}=\rho\left(i_{r_{1}}\right)
$$

and the incremental resistance matrix is $R_{1}\left(i_{r_{1}}\right)=\rho^{\prime}\left(i_{r_{1}}\right)$. Analogously, voltage-controlled resistors are governed by

$$
i_{r_{2}}=\gamma\left(v_{r_{2}}\right)
$$

and the incremental conductance matrix is $G_{2}\left(v_{r_{2}}\right)=\gamma^{\prime}\left(v_{r_{2}}\right)$. The only change in the proof of Theorem 1 involves the block $\left(-R\left(i_{r}\right) \quad I_{r}\right)$ in the $B(x)$ matrix (4), which must be split and rewritten in the form

$$
\left(\begin{array}{cccc}
-R_{1} & 0 & I_{r_{1}} & 0 \\
0 & I_{g_{2}} & 0 & -G_{2}
\end{array}\right)
$$

However, denoting $R_{2}=G_{2}^{-1}$ and premultiplying this block by the non-singular matrix

$$
\left(\begin{array}{cc}
I_{r_{1}} & 0 \\
0 & -R_{2}
\end{array}\right)
$$

an operation which does not affect the index, we confer the block the form $(-R I)$, and the proof of Theorem 1 is valid since $R$ is positive definite provided that so they are $R_{1}$ and $G_{2}$. The same reasoning applies in the presence of flux-controlled memristors. 


\subsection{Tree-based models}

Theorem 1 provides, in particular, a characterization of the tractability index of tree-based circuit models. The choice of a tree gives the circuit equations the form

$$
\begin{aligned}
C\left(v_{c}\right) v_{c}^{\prime} & =i_{c} \\
L\left(i_{l}\right) i_{l}^{\prime} & =v_{l} \\
q_{m}^{\prime} & =i_{m} \\
0 & =v_{m}-M\left(q_{m}\right) i_{m} \\
0 & =v_{r}-\rho\left(i_{r}\right) \\
0 & =v_{\mathrm{co}}+F v_{\mathrm{tr}} \\
0 & =i_{\mathrm{tr}}-F^{T} i_{\mathrm{co}},
\end{aligned}
$$

where $v_{\text {co }}$ and $v_{\mathrm{tr}}$ (resp. $i_{\text {co }}$ and $i_{\mathrm{tr}}$ ) stand for the link and twig voltages (resp. currents).

In practice, different settings lead to the choice of special trees; in the absence of VC-loops and IL-cutsets, the tree is usually a proper one, including all voltage sources and capacitors and neither current sources nor inductors. When VC-loops and/or IL-cutsets are present, it is common to work with a normal tree instead, that is, a tree including all voltage sources, the maximum possible number of capacitors, the minimum possible number of inductors and no current source. This stems from the work of Bashkow and Bryant $[2,6,7]$ and allows for an explicit characterization of the order of complexity (namely, the state dimension or dynamical degree of freedom) of the circuit.

Corollary 2. The index characterization in Theorem 1 also applies to the tree-based model (31), regardless of the actual choice of the tree.

This is a straightforward consequence of the fact that (31) is a particular instance of (1) with $B=\left(\begin{array}{ll}F & I_{\mathrm{co}}\end{array}\right), D=\left(I_{\mathrm{tr}}-F^{T}\right)$. Note, incidentally, that the assumptions allowing for the choice of a proper tree correspond to those in the index one case considered in item (i) of Theorem 1, whereas the normal tree framework is accommodated in the index two context of item (ii).

\section{$5 \quad$ Concluding remarks}

In this paper we have characterized the tractability index of so-called branch-oriented models of electrical and electronic circuits including memristors. Our approach is based on the use of certain graph-theoretic properties, and also on several results coming from projector theory, matrix analysis and matrix pencil theory. The present framework applies under strict passivity assumptions, and accommodates in particular circuit models arising from the choice of a spanning tree. The results should be helpful in future analyses of the dynamics of memristive circuits, including qualitative and numerical aspects. 


\section{References}

[1] B. Andrásfai, Graph Theory: Flows, Matrices, Adam Hilger, 1991.

[2] T. R. Bashkow, The A matrix, new network description, IRE Trans. Circuit Theory 4 (1957) 117-119.

[3] S. Benderli and T. A. Wey, On SPICE macromodelling of $\mathrm{TiO}_{2}$ memristors, Electronic Letters 45 (2009) 377-379.

[4] B. Bollobás, Modern Graph Theory, Springer-Verlag, 1998.

[5] K. E. Brenan, S. L. Campbell and L. R. Petzold, Numerical Solution of Initial-Value Problems in Differential-Algebraic Equations, SIAM, 1996.

[6] P. R. Bryant, The order of complexity of electrical networks, Proceedings of the IEE, Part C 106 (1959) 174-188.

[7] P. R. Bryant, The explicit form of Bashkow's A matrix, IRE Trans. Circuit Theory 9 (1962) 303-306.

[8] X. Chen, G. Wu and D. Bao, Resistive switching behavior of Pt $/ \mathrm{Mg}_{0.2} \mathrm{Zn}_{0.8} \mathrm{O} / \mathrm{Pt}$ devices for nonvolatile memory applications, Appl. Phys. Lett. 93 (2008) 093501.

[9] L. O. Chua, Memristor - The missing circuit element, IEEE Trans. Circuit Theory 18 (1971) 507-519.

[10] L. O. Chua, C. A. Desoer and E. S. Kuh, Linear and Nonlinear Circuits, McGraw-Hill, 1987.

[11] L. O. Chua and S. M. Kang, Memristive devices and systems, Proc. IEEE 64 (1976) 209-223.

[12] D. Estévez-Schwarz and C. Tischendorf, Structural analysis of electric circuits and consequences for MNA, Internat. J. Circuit Theory Appl. 28 (2000) 131-162.

[13] L. R. Foulds, Graph Theory Applications, Springer, 1992.

[14] F. R. Gantmacher, The Theory of Matrices, vols. 1 \& 2, Chelsea, 1959.

[15] E. Griepentrog and R. März, Differential-Algebraic Equations and Their Numerical Treatment, Teubner-Texte zur Mathematik 88, Leipzig, 1986.

[16] E. Griepentrog and R. März, Basic properties of some differential-algebraic equations, Z. Anal. Anwend. 8 (1989) 25-40.

[17] M. Günther and U. Feldmann, CAD-based electric-circuit modeling in industry. I: Mathematical structure and index of network equations, Surv. Math. Ind. 8 (1999) 97-129. 
[18] M. Günther and U. Feldmann, CAD-based electric-circuit modeling in industry. II: Impact of circuit configurations and parameters, Surv. Math. Ind. 8 (1999) 131-157.

[19] E. Hairer and G. Wanner, Solving Ordinary Differential Equations II: Stiff and Differential-Algebraic Problems, Springer-Verlag, 1996.

[20] M. Hasler and J. Neirynck, Nonlinear Circuits, Artech House, 1986.

[21] M. Itoh and L. O. Chua, Memristor oscillators, Intl. J. Bifurcation and Chaos 18 (2008) 3183-3206.

[22] M. Itoh and L. O. Chua, Memristor cellular automata and memristor discrete-time cellular neural networks, Internat. J. Bifurcation and Chaos 19 (2009) 3605-3656.

[23] P. Kunkel and V. Mehrmann, Differential-Algebraic Equations. Analysis and Numerical Solution, EMS, 2006.

[24] R. März, A matrix chain for analyzing differential algebraic equations, Preprint 162, Inst. Math., Humboldt University, Berlin, 1987.

[25] R. März, Differential algebraic equations anew, Appl. Numer. Math. 42 (2002) 315-335.

[26] R. März, The index of linear differential algebraic equations with properly stated leading terms, Results in Mathematics 42 (2002) 308-338.

[27] R. März, Differential algebraic systems with properly stated leading term and MNA equations, in Modeling, Simulation, and Optimization of Integrated Circuits (Oberwolfach, 2001), Int. Ser. Numer. Math. 146 (2003) 135-151.

[28] M. Messias, C. Nespoli and V. A. Botta, Hopf bifurcation from lines of equilibria without parameters in memristors oscillators, Internat. J. Bifurcation and Chaos 20 (2010) 437450 .

[29] B. Muthuswamy, Implementing memristor based chaotic circuits, Internat. J. Bifurcation and Chaos 20 (2010), in press, 2010.

[30] Y. V. Pershin and M. Di Ventra, Spin memristive systems: Spin memory effects in semiconductor spintronics, Physical Review B 78 (2008) 113309.

[31] P. J. Rabier and W. C. Rheinboldt, Theoretical and numerical analysis of differentialalgebraic equations, Handbook of Numerical Analysis, Vol. VIII, 183-540, North-Holland (2002).

[32] T. Reis, Circuit synthesis of passive descriptor systems - a modified nodal approach, Internat. J. Circuit Theory Appl. 38 (2010) 44-68. 
[33] G. Reiszig, The index of the standard circuit equations of passive RLCTG-networks does not exceed 2, Proc. ISCAS'98, vol. 3, 419-422, 1998.

[34] R. Riaza, Differential-Algebraic Systems. Analytical Aspects and Circuit Applications, World Scientific, 2008.

[35] R. Riaza, Nondegeneracy conditions for active memristive circuits, IEEE Trans. Circuits and Systems - II 57 (2010) 223-227.

[36] R. Riaza, Graph-theoretic characterization of bifurcation phenomena in electrical circuit dynamics, Internat. J. Bifurcation and Chaos 20 (2010) 451-465.

[37] R. Riaza and C. Tischendorf, Semistate models of electrical circuits including memristors, Internat. J. Circuit Theory Appl., accepted, in press, 2010.

[38] R. Riaza and C. Tischendorf, The hyperbolicity problem in electrical circuit theory, Mathematical Methods in the Applied Sciences, accepted, in press, 2010.

[39] G. S. Snider, Spike-timing-dependent learning in memristive nanodevices, Proc. IEEE Intl. Symp. Nanoscale Architectures 2008, pp. 85-92.

[40] D. B. Strukov, G. S. Snider, D. R. Stewart and R. S. Williams, The missing memristor found, Nature 453 (2008) 80-83.

[41] M. Takamatsu and S. Iwata, Index characterization of differential-algebraic equations in hybrid analysis for circuit simulation, Internat. J. Circuit Theory Appl. 38 (2010) 419-440.

[42] C. Tischendorf, Topological index calculation of DAEs in circuit simulation, Surv. Math. Ind. 8 (1999) 187-199.

[43] C. Tischendorf, Coupled systems of differential algebraic and partial differential equations in circuit and device simulation. Modeling and numerical analysis, Habilitationsschrift, Humboldt-Univ. Berlin, 2003.

[44] J. Vlach and K. Singhal, Computer Methods for Circuits Analysis and Design, Van Nostrand Reinhold ITP,

[45] X. Wang, Y. Chen, H. Xi, H. Li and D. Dimitrov, Spintronic memristor through spintorque-induced magnetization motion, IEEE Electron Device Letters 30 (2009) 294-297.

[46] J. J. Yang, M. D. Pickett, X. Li, D. A. A. Ohlberg, D. R. Stewart and R. S. Williams, Memristive switching mechanism for metal/oxide/metal nanodevices, Nature Nanotechnology 3 (2008) 429-433. 